\title{
RECURSOS Y COMPETITIVIDAD EN LAS SUB- RAMAS INDUSTRIALES DE ALIMENTOS Y BEBIDAS EN MÉXICO (2004, 2009 Y 2014)
}

\author{
ERIC ISRAEL RIOS NEQUIS \\ Universidad Autónoma de Baja California, México \\ eric.israel.rios.nequis@uabc.edu.mx \\ DANIELA EDITH CARBAJAL CANSINO \\ Universidad Politécnica Metropolitana de Hidalgo, México \\ decansino@outlook.com \\ MICHELLE EDITH HERNÁNDEZ SOLIS \\ Universidad Politécnica Metropolitana de Hidalgo, México \\ solismichelle350@gmail.com
}

\section{RESUMEN}

El trabajo de investigación abarca un análisis cuantitativo de los recursos con los que cuentan lassub-ramasindustriales dealimentosybebidas en México para generar ventas y disminuir competencia en sus respectivos ambientes competitivos para los periodos censales de 2004, 2009y 2014. La evidencia muestra que los saldos de inventarios iniciales son un recurso valioso porque inciden positivamente en las ventas y erosionan competencia, por lo que denotan, con ello, los elementos necesarios para ser una ventaja competitiva sostenible o superior.

PALABRAS CLAVE: VENTAJA COMPETITIVA, INVENTARIOS, VENTAS, BARRERAS A LA ENTRADA, ESTRATEGIAS.

\section{ABSTRACT}

The research work includes a quantitative analysis of the resources available for the food and beverages industrial sub-branches to generate sales and diminish competition in their respective competitive environments in Mexicofor the 2004, 2009 and 2014 periods according to the economic census in INEGI. The evidence shows that initial inventories balances are a valuable resource because it positively affects sales and erodes competition, thus denoting the necessary elements to be a sustainable or superior competitive advantage.

KEYWORDS: COMPETITIVE ADVANTAGE, INVENTORIES, SALES, ENTRY BARRIERS, STRATEGIES. 


\section{INTRODUCCIÓN}

El presente trabajo de investigación estudia el comportamiento de las ventajas competitivas, en términos de efectividad, mediante indicadores como ventas y rivalidad de la competencia (unidades económicas en el censo económico $|N E G|)$. De acuerdo con la teoría de la administración estratégica, las estrategias pueden generar ventaja competitiva, la cual se traduce en un desempeño superior respecto a la competencia.

Obtener una posición de ventaja competitiva y mejorar el desempeño de una empresa en relación con sus competidores son dos de los objetivos principales que las compañías deben trazarse por alcanzar. Para conseguir una ventaja competitiva que no solo coincida con la de los competidores de su empresa, sino que, también, supere los promedios de desempeño industrial, las organizaciones empresariales deben, primero, comprender la relación entre las fortalezas y debilidades internas de su organización, así como los posibles efectos en sus ventajas competitivas y en el rendimiento de la empresa (Ismail, Rose, Abdulla, y Uli, 2010).

La teoría de planeación estratégica es compatible con los planteamientos teóricos de la Organización Industrial (O.I.). La última aborda una correlación entre la ventaja competitiva y mayores ganancias, que resultan en mayor poder o concentración de mercado. Porter (1980) lo denomina rentabilidad, que es resultado de las estrategias y operaciones de negocio que generan valor.

Desde el punto de vista de Porter (1985), las estrategias de la compañía aplicadas en una industria pueden afectar el desempeño actual de un sector industrial (rivalidad entre competidores). Según el autor, una empresa no puede ser prisionera y sus acciones pueden cambiar las reglas de la competencia (Porter, 1985). El mismo analista argumenta que una estrategia sostenible requiere que una empresa tenga barreras implícitas para dificultar la imitación de la competencia (Porter, 1985).

La teoría de la Organización Industrial menciona que una barrera de entrada es una ventaja de los vendedores establecidos en una industria sobre los posibles vendedores entrantes. Esto se refleja en la medida en que los vendedores establecidos pueden elevar sus precios constantemente por encima de los niveles competitivos sin atraer nuevas firmas para ingresar a la industria (Bain, 1956).

Una barrera a la entrada es cualquier cosa que impida que un emprendedor cree instantáneamente una nueva empresa en un mercado. Una barrera de entrada a largo plazo es un costo necesariamente incurrido por un nuevo participante que los titulares no tienen (o no tuvieron que) soportar (Carlton y Perloff, 1994).

En síntesis, una ventaja competitiva debe ser evaluada mediante indicadores, ya que busca un desempeño superior por parte de las empresas. Al mismo tiempo, pueden afectar el entorno competitivo de la industria y, en algunos casos, podrían ser una barrera de entrada.

Diversos estudios empíricos en México han evaluado la concentración en ventas que hay en distintas industrias de Alimentos y Bebidas. Algunos, como el de Lef (1974), Bellon (1980, en Vázquez, 2015) y Rendon y Morales (2008), describen a las industrias de alimentos y bebidas, como conjunto de empresas, grandes, jurídicamente independientes, relacionadas por lazos familiares, financieros y comunidad de dirigentes (administración), donde se cuenta con una empresa controladora, estratega que organiza y administra las inversiones, las decisiones financieras que afectan el pago y reparto de utilidades y las transferencias de bienes entre empresas con el fin de lograr beneficios y ventajas para la totalidad del grupo (Rendon y Morales, 2008).

Vázquez (2015), por su parte, analiza la tendencia a la concentración de ventas en las industrias 
de alimentos y bebidas, ubicando un grupo de 30 empresas líderes en el ramo dentro del clasificado de las 500 empresas de Expansión para el periodo 2008, donde compañías como Bimbo (panadería), Maseca (maíz y tortillas), Nestlé (alimentos diversos), Sigma (alimentos diversos) y Bachoco (pollo) ostentaban el $24 \%$ de las ventas totales del grupo. Entre los elementos del análisis se destaca la logística y altas capacidades de distribución, la constante compra de empresas del mismo sector, el desarrollo de procesos de operación y negocios donde hay innovación tecnológica y la constante concentración hacia duopolios en industrias, como en el caso de las bebidas con Grupo Femsa y Pepsico en México o de tabaco para el caso de British American Tobaco y Philip Morris, que compraron las empresas mexicanas (Cigatam y Cigamod).

Otros trabajos como el de Ríos, Olvera y González (2016) analizan el oligopolio en la industria de los lácteos, donde se destaca la diferenciación de productos como patrón de competencia en dicha industria. Argumentan que Lala y Alpura tienen dominancia en dicha estrategia y que empresas como Santa Clara son una empresa de nicho que opera con precios altos, contradiciendo los supuestos clásicos de la economía, donde se asume que el líder de mercado es líder de precios.

En este sentido, se visualiza el dominio de Pepsi y Coca-Cola en el presente sector, aunque el refresco de cola es capaz de generar mayores ingresos vía precio, denotando la dominancia de dicha Coca-Cola en el país, pues obtiene más de $50 \%$ de la participación de mercado. Sin dejar de lado la capacidad de distribución de FEMSA (Ríos, López, y Garzón, 2016), o en la industria de los helados, donde la diferenciación de producto o las capacidades logísticas son elementos a considerar en la dominancia de Nestlé y Holanda en dicho mercado. En ese caso, empresas como Hérdez adquirieron la línea de helados de Nestlé y dicha sub-rama industrial gasta el doble en publicidad y promoción como porcentaje de sus ventas respecto a otras sub-ramas dentro de los productos lácteos (Ríos, Conraud, y Cansino, 2018).

Ante este fenómeno de competitividad, es prudente preguntarse ¿cuáles son los recursos económicos de las compañías que están asociados positivamente a las ventas como indicadores de ventaja competitiva en las sub-ramas industriales de alimentos y bebidas en México? Derivada de la problemática, se plantea una pregunta adicional: ¿Qué recursos de ventaja competitiva son monopólicos o están asociados al grado de competencia en las sub-ramas industriales de alimentos y bebidas en México?

El objetivo principal del artículo es encontrar los recursos de las compañías que están positivamente asociados a las ventas en las sub-ramas industriales de fabricación de alimentos y bebidas para los períodos 2004, 2009 y 2014 en México. Particularmente, este trabajo evalúa algunos factores que se correlacionan negativamente con el grado de competencia para los periodos mencionados.

La investigación intenta probar que recursos como los activos y requerimientos de capital están asociados positivamente a las ventas, clasificándose como ventajas competitivas pero la segunda variable es capaz de eliminar competidores, lo que la diferencia como ventaja competitiva sostenible en las sub-ramas industriales de alimentos y bebidas para los periodos 2004, 2009 y 2014 en México. En otras palabras, las empresas tienen capacidad de apalancamiento con sus clientes diversos debido a que los inventarios iniciales son fundamentales para permanecer en el negocio para las industrias mencionadas.

La hipótesis secundaria busca demostrar que los gastos publicitarios tienen efectos poco significativos como estrategia clave para aumentar ventas y poseen nula significancia en la reducción de la cantidad competidores para los sectores industriales mencionados; es decir, no son un buen 
indicador para detectar eliminación de competencia o un referente de barreras a la entrada.

El artículo está compuesto de tres secciones. La primera trata de una revisión literaria del problema de investigación. La segunda sección está integrada por la metodología y los resultados que responderán la hipótesis de investigación. Finalmente, el tercer apartado presenta las conclusiones del trabajo de investigación.

\section{CONTEXTO TEÓRICO}

La presente sección presenta los aportes de dos visiones respecto a la búsqueda de la ventaja competitiva. Una es el paradigma de estructura-conducta y desempeño (ECD) en la teoría de la organización industrial. La segunda es la visión basada en recursos. Dentro de la teoría de la planeación estratégica se ubica a Michael Porter como uno de los exponentes del primer paradigma mencionado (Ma, 2000; Bridoux, 2004), y en el segundo paradigma a teóricos como Wernefelt (1984), Rumelt (1984) y Barney (1986; 1991). Las dos posturas intentan explicar las razones por las que una empresa tiene un desempeño superior frente a las demás. El objetivo de los apartados consiste en desagregar los indicadores que evalúan a la ventaja competitiva y dimensionan el desempeño superior.

\section{El paradigma estructura-conducta- desempeño}

La idea central del paradigma estructura, conducta y desempeño (ECD) tiene sustento en la teoría de la economía industrial (organización industrial), la cual menciona que una sólida posición de mercado y defendible en una industria atractiva recae en una ventaja competitiva sostenible (Porter, 1980; 1985; Ma, 2000). Mediante el análisis de las fuerzas, Porter (1980) ilustra que la sostenibilidad de las utilidades o ganancias es lograda por la negociación y la forma de administrar la competencia directa e indirecta (Porter, 1991). El analista indica que las utilidades sostenibles dependen de una ventaja sostenible (Porter, 1980; 1985).

Es importante mencionar que una ventaja competitiva tiene elementos asociados como la posibilidad de afectar la entrada de futuros entrantes, ya que se busca obtener utilidades constantes y superiores a la competencia. Desde la perspectiva de Porter (1980), la función de la empresa consiste en formular una estrategia competitiva para alcanzar un desempeño económico superior, donde el primer elemento es capaz de cambiar las reglas de la industria en favor de la compañía. Es decir, la empresa puede elegir las estrategias que disuadan o dificulten la entrada en los sectores a los que pertenecen.

En el enfoque de Porter no se atribuye única o mayoritariamente las fuentes de las ganancias en las capacidades de la empresa; sino a la naturaleza y el balance de las cinco fuerzas competitivas (Schoemaker, 1990). En este contexto, Porter afirma que para alcanzar ganancias sostenibles es necesario tener ventajas competitivas sostenibles (Porter, 1991). Esto permite preguntarse: ¿Cómo se crea la ventaja competitiva? Las siguientes líneas contestan dicha interrogante.

La ventaja competitiva crece fundamentalmente a partir del valor que una empresa puede crear para sus compradores y que excede el costo de su creación (Porter, 1985). Asimismo, para Porter, el valor es lo que los consumidores esperan pagar y el valor superior comienza con ofrecer precios más bajos que los competidores por beneficios equivalentes. Otra opción es ofrecer beneficios únicos a precios más altos. Esto es el resultado de obtener ventajas competitivas vía liderazgo en costos y por diferenciación (Porter, 1985).

Respecto a los planteamientos de Porter, una importante cuestión surge de reflexionar si la ventaja en costos y la diferenciación son suficientes, así como necesarias, para un desempeño superior 
frente a la competencia (Ma, 2000). Si la respuesta anterior es negativa, se debe concluir que la ventaja competitiva explicada por Porter (1980; 1985) no es equiparable en su totalidad al desempeño. Entre diversos ejemplos, analistas como Ma (2000) argumentan que hay empresas respaldadas por sus gobiernos locales para obtener ventajas y no necesariamente sus ganancias dependerán de un liderazgo en precios o de la diferenciación.

Las ventajas competitivas no necesariamente deben ser atribuidas a los últimos elementos en el párrafo anterior, puesto que no son los únicos determinantes en el desempeño de las empresas. El desempeño superior puede ser asociado a la velocidad (Stalk, 1990; Eisendhardt y Brown, 1998) o la flexibilidad (Sánchez, 1993; 1995). Para Ma (2000) es la combinación de múltiples ventajas competitivas. Contrariamente, hay evidencia empírica que analiza el comportamiento del desempeño superior, el cual emana en mayor medida de las capacidades internas de las empresas que del entorno competitivo (Schmalensee, 1985; Rumelt, 1991).

\section{La visión basada en recursos}

Como teoría alterna, surge la perspectiva basada en recursos (RBV), la cual explica que los recursos únicos de las empresas son fuentes de ventajas competitivas sostenibles (Barney, 1991). Para generar dicha ventaja competitiva, el recurso debe ser raro, valioso, no imitable, no comerciado e insustituible. Asimismo, la esencia de la compañía en específico (Barney, 1986, 1991; Dierickx y Cool, 1989; Grant, 1991).

En esta corriente, hay trabajos notables e integrativos como el de Peteraf (1993), el cual resume las piedras angulares de la ventaja competitiva de la visión basada en recursos. Para el autor, hay heterogeneidad en la dotación de recursos que generan ventajas competitivas, asumiendo la limitante de la competencia para sostener la rentabilidad. De hecho, la movilidad imperfecta de recursos genera las ganancias dentro de la empresa. Este último elemento es resultado de que los recursos no pueden ser comerciados fácilmente en el mercado de factores; por consecuencia, son difíciles de acumular e imitar (Bridoux, 2004).

Bajo este enfoque, el recurso único de la empresa es tratado y relacionado inherentemente con el desempeño, ya que es poco imitable, único e inmóvil y se somete a una evaluación asociada a la rentabilidad económica (Barney, 1991). En este sentido, se encuentra la asociación entre ventaja competitiva (recursos únicos) y desempeño (rentabilidad económica). Para Ma (2000), esta correlación es más directa que la presentada por Porter (1980) porque no se tiene que clasificar únicamente a la ventaja competitiva por liderazgo costos o diferenciación. Si una empresa posee recursos poco o nulamente imitables, raros y valiosos, necesariamente originan un desempeño superior; es decir, para el autor mencionado la ventaja competitiva consecuentemente ocasiona un desempeño superior.

La visión basada en recursos ha desarrollado un análisis cauteloso respecto a algunos fenómenos. Entre ellos, se pueden mencioar la economía de la compresión del tiempo, eficiencia en masa de activos (Dierickx y Cool, 1989), los activos intangibles que incluyen elementos como la información (Sampler, 1998), el conocimiento (Spender, 1996) y capacidades dinámicas (Teece, Pisano, y Shuen, 1997).

A pesar de lo anterior, la visión basada en recursos tiene un problema en su análisis, ya que no ha logrado integrar los recursos con el medio ambiente de la compañía. Lo anterior quiere decir que los teóricos no explican la forma en que los recursos se comportan en el ambiente empresarial (Bridoux, 2004). En este sentido, la última autora citada expone un modelo donde incorpora los recursos y capacidades de las empresas al medio ambiente competitivo de la empresa. 
Por otro lado, la visión basada en recursos carece de una unidad propia de análisis para estudiar la ventaja competitiva porque solo toma en cuenta que un recurso individual es capaz de generar ventaja competitiva. Sin embargo, no explica si este recurso está agrupado con otros, no responde la teoría a cuestiones como la forma en que interactúan, si estos trabajan en conjunto con otros y su sinergia dentro de un sistema (Foss, 1998).

El marco teórico indica que la ventaja competitiva tiene dos maneras de ser abordada en su explicación. Sin embargo, ambas posturas (Visión Basada en Recursos y Enfoque ECD) tienen limitantes en su análisis. La teoría de Porter, aun cuando afirma que la ventaja competitiva está asociada al liderazgo en precios y diferenciación, indirectamente señala que la eliminación de competidores es fundamental para permanecer en el mercado. Es decir, las barreras a la entrada son fundamentales para dominar la industria. Este planteamiento se desarrollará en la siguiente sección.

\section{La ventaja competitiva: forma de evaluar su desempeño y su modelación teórica}

La literatura aborda dos formas de evaluar el desempeño de la ventaja competitiva. Una está relacionada con las finanzas y la rentabilidad, mientras la otra es de orden no financiero. Los indicadores de la primera están basados en las ventas y en los retornos sobre activos y su par en los procesos organizacionales.

Los trabajos que utilizan las ventas como instrumento de evaluación de ventaja competitiva aplican indicadores como nivel de ingresos por las ventas, rentabilidad, retorno de la inversión, productividad, valor agregado, cuota de mercado y crecimiento del producto (Wang y Lo, 2003; Neely, 2005; Falsaw, Glaister y Ekrem, 2006). Otras investigaciones evalúan a la ventaja competitiva con la eficiencia organizacional, cuando esta es medida con base en la eficiencia como procesos internos, satisfacción del cliente, desarrollo de carrera profesional y satisfacción en el trabajo (Wang y Lo, 2003; Neely, 2005).

Finalmente, se han utilizado variables mediadoras para explicar de manera profunda el desempeño de la ventaja competitiva. Ketokivi y Schroeder (2004), Morgan, Kaleka y Katsikeas (2004) y Ainuddin, Beamish, Hulland y Rouse (2007) establecen que la edad o experiencia de la empresa y su tamaño son factores que moderan o controlan, en cierta medida, el desempeño de la ventaja competitiva. Para unos, la edad de las empresas es categorizada en plantas viejas y nuevas (Ketokivi y Schroeder, 2004). Para otros, es la cantidad de años que lleva la empresa exportando sus productos (Morgan et al., 2004) y algunos consideran el tiempo que tiene una compañía involucrada en un Joint-Venture (Ainuddin et al., 2007). En síntesis, los trabajos indican una hipótesis inicial que consiste en relacionar la edad de las empresas con un mejor desempeño que sus pares jóvenes.

Otro tema de debate tiene relación con el tamaño de la empresa como mediadora. Hay estudios que encuentran significancia de la variable en la asociación entre la ventaja competitiva y su desempeño. Esta es medida por la cantidad de empleados (Ketokivi y Schroeder, 2004) o por el número de empleados de tiempo completo (Morgan et al., 2004). Por otro lado, hay evidencia que no encuentra una significancia en el fenómeno, tomando como referencia la cantidad de la fuerza laboral involucrada en el Joint-Venture (Ainuddin et al., 2007).

En un estudio reciente, Ismail et al. (2010) reafirman que hay una correlación entre ventaja competitiva y desempeño, destacando que la edad puede ser un elemento mediador en la asociación indicada. Sus resultados y conclusiones radican en que la experiencia medida por su edad ayuda a que las empresas puedan ser eficientes en sus operaciones, generando un mejor desempeño que sus pares más jóvenes (curva de la 
experiencia). Los autores contribuyen al debate suscitado por el tamaño de la empresa, ya que esta no tiene significancia en el desempeño. Su explicación radica en la intervención de rápidos cambios tecnológicos, la subcontratación (outsourcing) y la globalización, los cuales inhiben la influencia de dicha variable moderadora.

\section{REVISIÓN DE MODELOS TEÓRICOS}

La revisión literaria conlleva a una asociación lineal entre ventaja competitiva y desempeño organizacional, el cual puede ser evaluado por retornos sobre activos y ventas (Figura 1).

La visión basada en recursos postula, con autores como Barney (1991) y Newbert (2007), que los recursos generan una ventaja competitiva, son raros, poco imitables y ocasionan un desempeño dado. No obstante, Raduan, Jegak, Haslinda y Alimin (2009) diferencian que los recursos no imitados pueden detonar una ventaja competitiva sostenible y, a su vez, un desempeño sostenible (Figura 2). Este análisis enfatiza que el recurso es único; por tanto, es superior a los que posee la competencia, lo que otorga dominancia frente a los demás.

La ventaja competitiva sostenible debe cumplir con tres recursos genéricos como los activos, capacidades de producción, acceso a canales de distribución y suministro, conocimiento, competencia y eficiencia en las operaciones de negocios (Ma, 1999). Para el último autor citado, las empresas deben ir más allá con los recursos genéricos, puesto que los rivales intentan adelantarse con la ejecución de dichos recursos de manera proactiva o preventiva (Ma, 1999).

Finalmente, la literatura aporta un modelo amplio e híbrido, el cual retoma las fuerzas competitivas de Porter y las capacidades o recursos internos de la empresa que contribuyen a la generación de la estrategia (comportamiento competitivo). Este último denotando la ventaja competitiva sostenible, la cual es evaluada por su desempeño (Figura 3).

Bajo este contexto, se contempla que hay un flujo regresivo entre las variables involucradas en el modelo. Los recursos y el ambiente competitivo influyen en la estrategia; es decir, hay elementos internos y externos que son base para la generación de la estrategia, la cual tiene correlación directa con la ventaja competitiva sostenible y esta, a su vez, con el desempeño, el cual va a retroalimentar a los recursos internos de la empresa y al ambiente competitivo (fuerzas competitivas de Porter).

En este sentido, el modelo Bridoux (2004) contiene elementos desagregados que están involucrados en la creación o formulación de la ventaja competitiva. Sin embargo, no aborda directamente la temática relacionada con el poder de monopolio, ya que la estrategia cuando es competitiva, tiene capacidad para erosionar la competencia. Al respecto, Porter (1980) clasifica seis recursos de barreras a la entrada. De ellas, tres permanecen asociadas al liderazgo en costos y diferenciación: i) economías de escala (costos), ii) diferenciación de producto (diferenciación), iii) costos de cambio (costos).

Sin embargo, hay tres que son recursos: uno de ellos son los accesos a canales de distribución (que se mencionó anteriormente en la visión basada en recursos); otra es el requerimiento de capital (recursos); la última refiere a desventajas en costos no relacionadas con la economía de escala, como la propiedad de alguna tecnología, acceso favorable a materias primas, localización o ubicación favorable, subsidios gubernamentales y la curva de aprendizaje. Como se puede apreciar, las barreras a la entrada son una combinación de la estrategias y de los recursos, los cuales pueden no ser imitados, tal y como lo establece la teoría mencionada anteriormente.

Este artículo propone que la ventaja competitiva debe ser evaluada bajo un indicador que es 
FIGURA 1

VARIABLES E INDICADORES ENVUELTOS EN EL ANÁLISIS DE LA VENTAJA COMPETITIVA

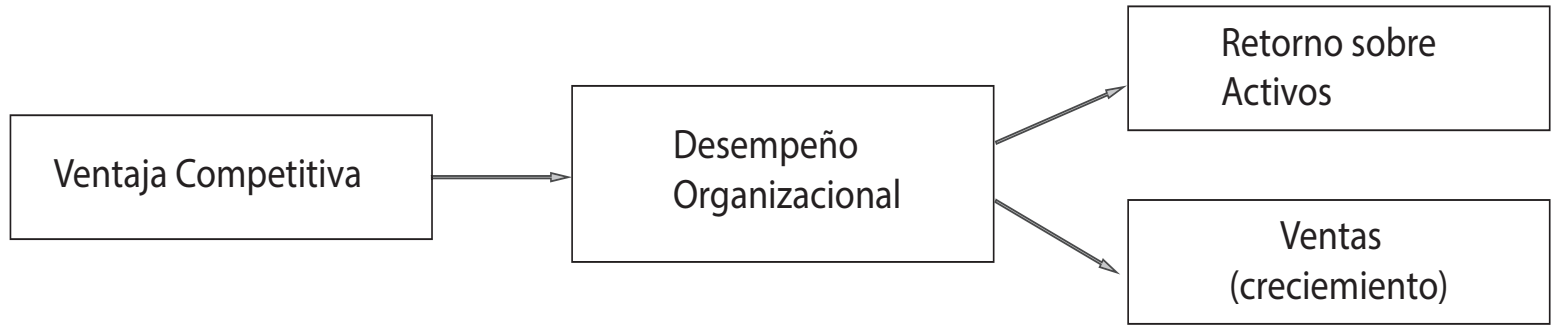

Fuente: Majeed (2011)

FIGURA 2

CARACTERÍSTICAS DE LOS RECURSOS QUE DETONAN VENTAJA COMPETITIVA Y DESEMPEÑO

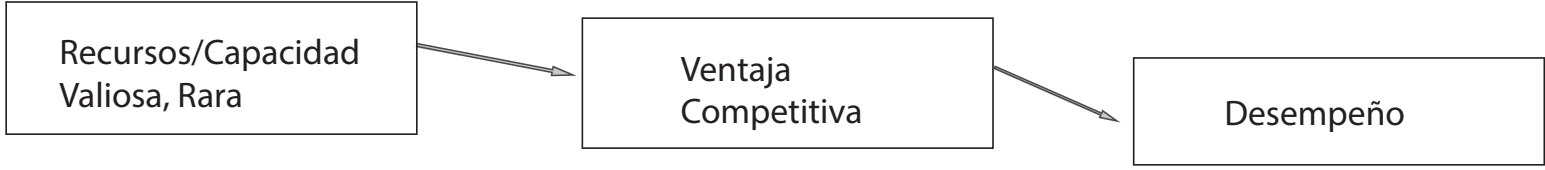

Recurso/Capacidad

No imitable, valioso

insustituible, raro

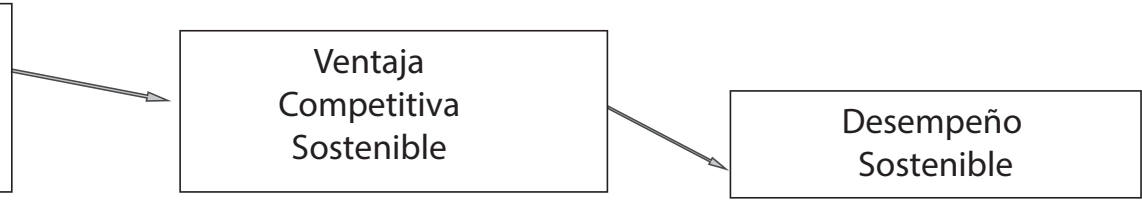

Fuente: Raduan, Jegak, Haslinda y Alimin (2009).

FIGURA 3

ELEMENTOS QUE INTEGRAN LA VENTAJA COMPETITIVA DESDE UNA PERSPECTIVA INTEGRAL (RECURSOS INTERNOS Y AMBIENTE COMPETITIVO)

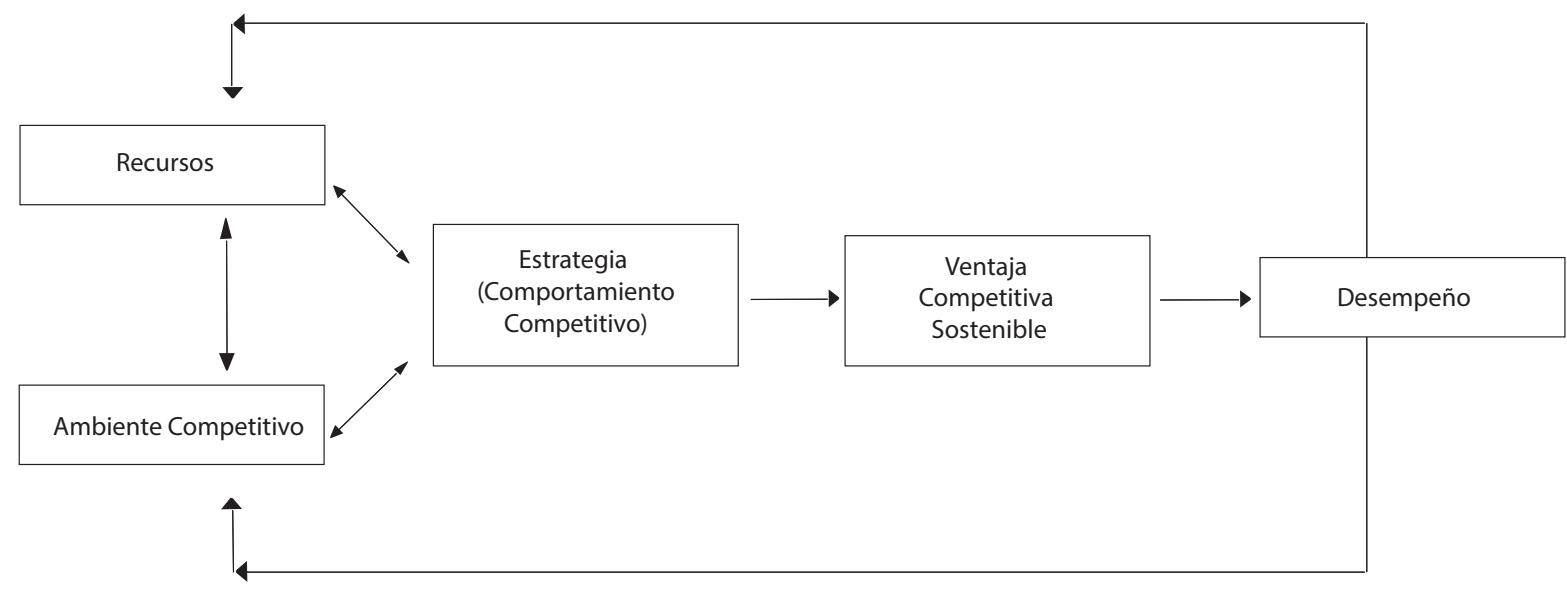

Fuente: Bridoux (2004). 
poco aplicado en la evidencia empírica pero que es mencionado de manera indirecta, este es la cantidad de competidores. Esta investigación propone a las barreras a la entrada como indicadores alterno a las ventas para determinar si la ventaja competitiva es sostenible o no.

En las seis fuentes de barreras a la entrada se ubican indicadores que se rescatan para la estimación econométrica. Estos son economías de escala en producción, marketing e investigación. Para la diferenciación de producto, se ubica a los efectos ganados por publicidad, atención a clientes, diferencias de producto o, simplemente, ser el primero en llegar a la industria. En requerimientos de capital, está el gasto publicitario, gastos en investigación y desarrollo (i+d), capacidad de otorgar crédito a los clientes, inventarios y capacidad para cubrir pérdidas relacionadas con el inicio de negocios; es decir, capacidad de apalancamiento. Los costos de cambio son aquellos relacionados con cualquier cambio en las operaciones y estructura de la empresa: proveedores, entrenamiento o capacitación de empleados, rediseño de productos, costo de nuevos equipos auxiliares. En acceso a nuevos canales de distribución se ubican los gastos de publicidad cooperativa con el canal y descuentos.

El trabajo plantea que los recursos inciden directamente en las ventas. Este último actúa como indicador de desempeño en las sub-ramas industriales de alimentos y bebidas. Sin embargo, la ventaja competitiva sostenible genera ventas y disminuye competencia (Figura 4). Esto tiene fundamento en Porter (1980), porque su análisis radica en el poder de mercado y los beneficios o rentas monopólicas (Conner, 1991). La ventaja competitiva de Porter (1980) se deriva de obstaculizar las fuerzas competitivas que tienden a conducir rendimientos económicos cercanos a cero, construyendo barreras de entrada y restringiendo el suministro (Bridoux, 2004).

Para Michael Porter, la ventaja competitiva se deriva de impedir el ingreso de fuerzas competitivas que ocasionan rendimientos cercanos a cero mediante barreras a la entrada y movilidad que restrinjan la oferta (Porter, 1980; Bridoux, 2004). En este contexto, Williamson (1991) confirma los

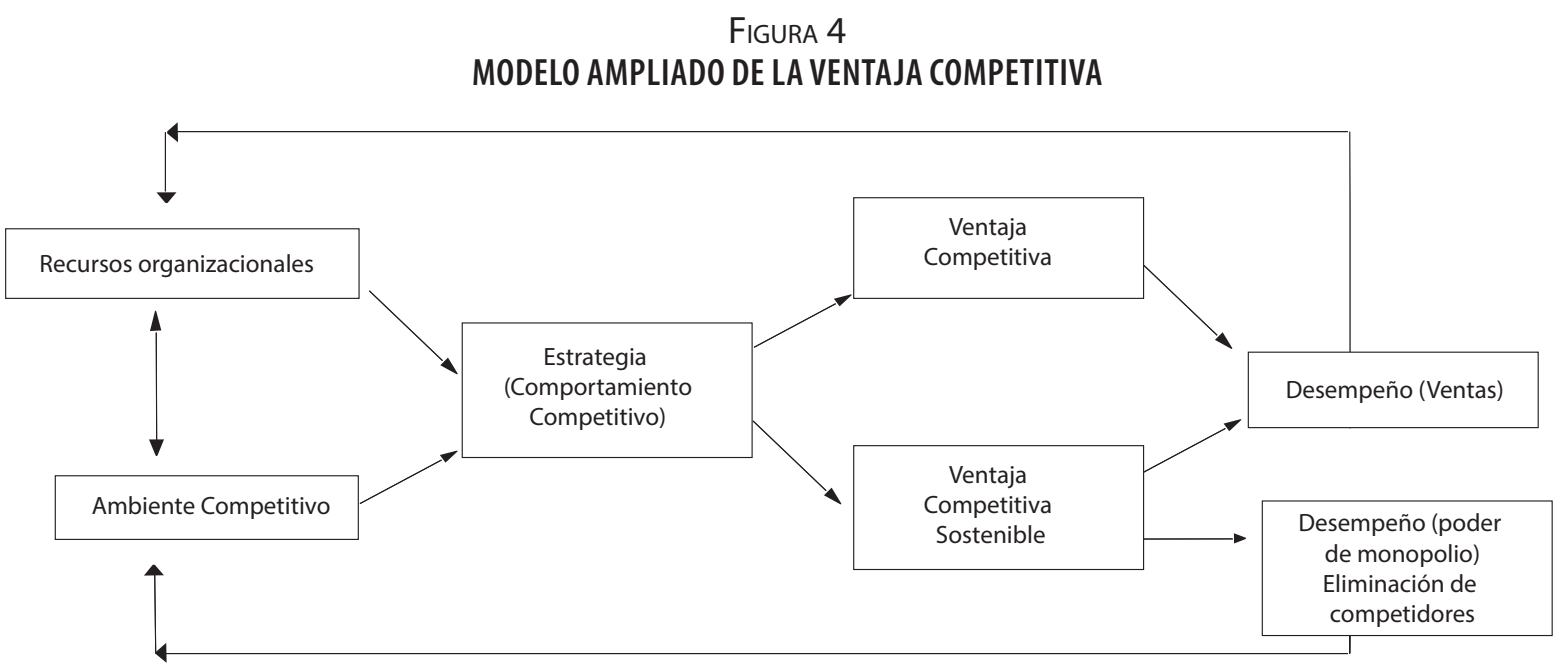

Fuente: Elaboración propia con planteamientos de Bridoux (2004). 
planteamientos del poder de mercado y los esfuerzos estratégicos para mitigar la competencia. Según el autor, la eficiencia es fundamental para las empresas que tienen poder de mercado, las cuales son un pequeño grupo del total y porque un esfuerzo de estrategia raramente prevalecerá si un programa de operaciones y negocios está orientado con excesos de costos significativos en producción, distribución u organización.

Por su parte, Grant (1991) confirma y afina los postulados, argumentando que el poder de mercado y las rentas monopólicas dependen de los recursos empresariales. Para el autor, un requisito previo de poder de mercado es la presencia de barreras a la entrada, las cuales tienen soporte en las economías de escala, patentes, ventaja en la experiencia, reputación de marca y otros recursos que poseen las empresas competidoras pero que los entrantes potenciales adquirirán lentamente a costos elevados. Bajo este contexto, se propone un modelo teórico para distinguir una ventaja competitiva sostenible de una ventaja competitiva más simple, mediante una variable moderadora (grado de competencia).

La literatura ofrece una amplia explicación respecto a los beneficios que puede generar la ventaja competitiva en una empresa. Los debates se centran en las cualidades que deben tener una ventaja competitiva y su homóloga sostenible, sus métodos de evaluación (ventas y retorno sobre activos) y sus variables moderadoras (tamaño de la empresa y edad). Este trabajo de investigación propone añadir que las barreras a la entrada no son solo recursos o elementos asociados a las ventajas competitivas que impiden la entrada; es decir, las barreras están asociadas a un desempeño superior mediante ventas al trabajar con la estrategia competitiva de manera simultánea y así lograr dominancia en su sector industrial.

Poco se ha evaluado a la ventaja competitiva mediante el poder de monopolio, el cual pudiera ser una barrera a la entrada porque este último indicador ayuda a establecer un parámetro y condición para la entrada de competidores potenciales que buscan ingresar a un mercado donde hay rivalidad y que su intensidad es variable respecto a las formas en que se organiza cada industria. Es decir, las ventas y el grado de competencia ayudan a dimensionar la efectividad de una ventaja competitiva y el poder de mercado que puede emanar del comportamiento estratégico, razón por la cual el articulo considera, en cierta medida, que la estructura de mercado condiciona una conducta y esta su desempeño; a su vez, este último retroalimenta a su estructura. Esto se basa en nuevos enfoques de organización industrial ECD (Tarziján y Paredes, 2006).

La figura 4 es un modelo ampliado de elaboración propia que toma como referencia el planteado por Bridoux (2004) y concuerda con sus postulados porque los recursos organizacionales y el ambiente competitivo intervienen en la generación de la estrategia y esta puede ocasionar dos vertientes que no son incluidas en el modelo general (Figura 3); una es la ventaja competitiva tradicional, la cual genera ventas pero puede ser imitable en el corto o mediano plazo por las empresas seguidoras. La ventaja competitiva sostenible dura en el largo plazo porque obstaculiza la entrada de competidores y, además, genera dividendos para la compañía, aumentando con ello poder de mercado, rescatando la teoría de Porter (Figura 4).

El modelo es una extensión del planteado por Bridoux (2004) y retoma la relación regresiva entre ventas y recursos porque hay una especie de generación de dinero para reinvertir en recursos. Asimismo, con el crecimiento de ventas se gana espacio en la cuota de mercado y por medio de las barreras a la entrada se dificulta la competencia, cambiando el medio ambiente competitivo, concluyendo que la ventaja competitiva no necesariamente es explicada por un paradigma es una estrategia que se fusiona con diversos recursos tangibles e intangibles. 


\section{METODOLOGÍA Y RESULTADOS}

El estudio es cuantitativo y de alcance correlacional, con datos del censo económico que es registrado por el Instituto Nacional de Geografía y Estadística (INEGI). El total de la muestra abarca 52 sub-ramas industriales para el periodo 2014; 53 para 2009 y 50 en 2004. De este modo, la clasificación cambia con los periodos censales, razón por la cual se eligieron los modelos de regresión de corte transversal como herramienta de análisis. La muestra comprende 10 sub-ramas de bebidas y 42 alimentos para el año 2014, mientras en 2009 se registraron 11 y 43 para cada caso. Finalmente, la muestra se conformó de 9 y 41 respectivamente en 2004. Las variables que componen el modelo son numéricas, discontinuas y fueron transformadas a logaritmos para su evaluación (relación loglog), salvo las variables de rivalidad en la industria que son dummy, estas discriminan las sub-ramas que pertenecen a alimentos (1) de las que no (0) y para el caso de bebidas el mismo sentido: (1) pertenecen y $(0)$ no pertenecen.

Las variables fueron tomadas de la literatura porque, para Porter (1985), los requerimientos de capital son catalogados en la capacidad de otorgar créditos a los clientes y para cubrir pérdidas por un tiempo o tener amplios inventarios para trabajar. La variable inventarios fue tomada de dicha postura teórica. Asimismo, se toman los activos fijos totales porque, según la clasificación del INEGI (2014), estos tienen la capacidad de producir o proporcionar las condiciones necesarias para la generación de bienes y servicios, lo que permite deducir la presencia de economías de escala y de requerimientos de capital. Para algunos analistas de la visión basada en recursos, los activos son parte integral de la ventaja competitiva (Barney, 1991; 2002). Empíricamente para Morgan et al. (2004) y Ainnudin et al. (2007), recursos físicos, como la capacidad de la planta, maquinaria, equipo, producción y la tecnología contribuyen positivamente en un desempeño superior.
Por otro lado, se incluye otra variable (gastos de comunicación) que es una partida, donde la unidad económica (empresa) erogó gastos relacionados con los servicios utilizados para comunicarse, como el teléfono, fax, internet y otras tecnologías para operar. Esta partida puede ser vista como un recurso interno de la organización. Para Barney (2002), los recursos incluyen todos los activos, capacidades y procesos organizacionales que la empresa concibe para implementar estrategias que mejoren su eficiencia y su efectividad. Dentro de la partida de gastos, la diferenciación de productos es fundamental y los gastos publicitarios son un instrumento para diferenciar productos, así como un requerimiento de capital (Porter, 1985).

Otra variable no menos importante es la de intensidad de la rivalidad en la industria. El trabajo pretende explorar con esta aportación que el medio ambiente competitivo de las industrias de alimentos y bebidas es distinto. Por ende, pudiera tener una repercusión en el comportamiento de las ventas, por lo que se construye una variable ficticia o dummy que discrimina a las industrias de alimentos de sus homólogas de bebidas. Este planteamiento tiene fundamento en Porter (1985), porque el modelo de las cinco fuerzas centra su atención en la rivalidad de la industria, y en Bridoux (2004), quien asume que el ambiente competitivo condiciona la estrategia y esta, a su vez, genera un desempeño, lo que permite plantear que acorde al medio ambiente competitivo las ventas pudieran variar.

La investigación es relevante porque diversos trabajos se han centrado en realizar cuestionarios a ejecutivos de diversos sectores industriales en contextos locales e internacionales, los cuales intentan identificar los elementos que detonan la ventaja competitiva y su relación con el desempeño (Giménez y Ventura, 2002; Ray, Barney, y Muhanna, 2004; Morgan et al., 2004; Ainuddin et al., 2007; Ismail, et al., 2010). De hecho, hay metodologías teóricas que respaldan los trabajos 
previos (Sekaran, 2005). La propuesta recae en que se utiliza información censal con datos numéricos de cada sub-rama industrial de alimentos y bebidas, buscando respaldar los hallazgos de la evidencia empírica con datos generales. Principalmente, se pretende encontrar los recursos tangibles que se asocian a las ventas y eliminan competencia. A diferencia de los otros trabajos, se utiliza información oficial y no interviene las percepciones personales que puede tener un cuestionario.

El trabajo se compone de seis modelos econométricos; tres para analizar el efecto generado de las variables que son parte de los recursos internos de las empresas (transformadas en logaritmos) en las ventas y estos mismos sobre las unidades económicas (grado de competencia). La finalidad es discriminar el tipo de asociación de las ventajas competitivas (ventas) respecto a las sostenibles (poder de mercado mediante eliminación de competidores).

Como se mencionó, las variables dummy, la cual discrimina las sub-ramas que conforman alimentos y bebidas. Los efectos porcentuales en la regresión se explicarán en la próximas tablas y su demostración matemática es presentada por (Greene, 2012) que viene en la sección de Anexos.

Las especificaciones de los modelos son las siguientes:

$$
\begin{aligned}
& L_{n} \text { ventas }=\beta 0+\beta 1 L_{n} \text { Total de Inventarios } \\
& \text { Iniciales }+\beta 2 L_{n} \text { Activos Fijos Totales }+\beta 3 \mathrm{~L} \text { - } \\
& { }_{n} \text { Gastos Publicitarios }+\beta 4 L_{n} \text { Gastos de } \\
& \text { Comunicación+ } \beta 5 L_{n} \text { Rivalidad de la } \\
& \text { Industria+ } \varepsilon \\
& L_{n} u e=\beta 0+\beta 1 L_{n} \text { Total de Inventarios } \\
& \text { Iniciales }+\beta 2 L_{n} \text { Activos Fijos Totales }+\beta 3 \mathrm{~L}- \\
& { }_{n} \text { Gastos Publicitarios }+\beta 4 L_{n} \text { Gastos de } \\
& \text { Comunicación+ } \beta 5 L_{n} \text { Rivalidad de la } \\
& \text { Industria+ } \varepsilon
\end{aligned}
$$

Los resultados para los modelos que miden los efectos de las variables respecto a las ventas, poseen un coeficiente determinación de .9726, .9385 y .9200 para 2014, 2009 y 2004 respectivamente. Es decir el $97.26 \%, 93.85 \%$ y $92 \%$ de la variabilidad de las ventas son explicadas por las variables que integran los modelos de corte transversal. Las pruebas estadísticas fueron satisfactorias en la mayoría de las regresiones (homocedasticidad, normalidad, colinealidad y especificación), salvo la homocedasticidad para la regresión que tiene a unidades económicas como variable dependiente y corresponde al año 2014 (revisar Anexo).

La partida de inventarios iniciales fue una de las que obtuvo una significancia constante respecto a las ventas. Sus efectos indican que al aumentar en $1 \%$ los saldos de inventario inicial en libros, las ventas aumentarán en $45.84 \%$, 31.30\% y $23.69 \%$ para los periodos 2014, 2009 y 2004. Por su parte, los efectos del total de activos fijos indicaron que, al aumentar en $1 \%$ el total de dicha partida, las ventas crecerán en 36.35\%, 55.54\% y $46.47 \%$ cada una para 2014, 2009 y 2004 (tabla 1).

Otras variables no fueron homogéneas en cuanto a su significancia, tales como los gastos de comunicación, los cuales indican que al aumentar la cuenta en 1\%, las ventas ascenderán en $20.97 \%$ y $27.99 \%$ para 2014 y 2004. En este sentido, el medio ambiente competitivo para las industrias que pertenecen a la sub-rama de alimentos obtuvieron ventas mayores de 42.56 y 93.05 para los últimos dos periodos censales (2014 y 2009)1. Finalmente, se ubica a los gastos publicitarios, los cuales fueron significativos en 2009 y su efecto marginal indica que al aumentar en $1 \%$ los gastos publicitarios, estos ocasionan un incremento de 4.25\% (tabla 1).

Los resultados sobre el grado de competencia (unidades económicas) fueron interesantes. La $r$

'Revisar Anexo para ver el tratamiento de la variable dummy el cálculo de sus efectos porcentuales. 
TABLA 1

RESULTADOS DEL MODELO ECONOMÉTRICO (2004, 2009 Y 2014)

\begin{tabular}{|c|c|c|c|c|c|c|c|c|c|c|c|c|}
\hline Obs. & 51 & & R cuad. & 0.9726 & Obs.52 & & R cuad. & 0.9385 & 50 & & R cuad. & 0.9200 \\
\hline Ventas & Coef & $T$ & $p>t$ & Efecto \% & Coef & $\mathrm{T}$ & $p>t$ & Efecto \% & Coef & $\mathrm{T}$ & $p>t$ & Efecto \% \\
\hline Inv. Inic. & .4518 & 7.84 & 0.000 & 45.84 & .3130 & 4.50 & 0.000 & 31.30 & .2369 & 3.28 & 0.002 & 23.69 \\
\hline Act.Fij & .3635 & 4.65 & 0.000 & 36.35 & .5554 & 6.24 & 0.000 & 55.54 & .4647 & 4.79 & 0.000 & 46.47 \\
\hline Gastos Com. & .2097 & 3.71 & 0.001 & 20.97 & -.0007 & -0.01 & 0.993 & & .2799 & 2.32 & 0.025 & 27.99 \\
\hline Gastos Pub & -.0035 & -0.11 & 0.916 & - & .0425 & 2.39 & 0.021 & 4.25 & .0099 & 0.22 & 0.831 & - \\
\hline Alim. & .3546 & 2.44 & 0.019 & 42.56 & .6578 & 3.55 & 0.001 & 93.05 & .2679 & 1.44 & 0.157 & - \\
\hline
\end{tabular}

Fuente: Elaboración propia con Software Stata

TABLA 2

RESULTADOS DEL MODELO ECONOMÉTRICO SOBRE UNIDADES ECONÓMICAS $(2004,2009$ Y 2014)

\begin{tabular}{|c|c|c|c|c|c|c|c|c|c|c|c|c|}
\hline Obs. & 52 & & R cuad. & 0.3602 & Obs. 52 & & R cuad. & 0.6620 & 50 & & R cuad. & 0.5313 \\
\hline Uec. & Coef & $\mathrm{T}$ & $p>t$ & Efecto \% & Coef & $\mathrm{T}$ & $p>t$ & Efecto \% & Coef & $\mathrm{T}$ & $p>t$ & Efecto \% \\
\hline Inv. In. & -.9033 & -2.97 & 0.005 & -90.33 & -.8822 & -4.63 & 0.000 & -88.22 & -.5149 & -2.42 & 0.020 & -51.49 \\
\hline Act. Fij. & .9823 & 2.38 & 0.022 & 98.23 & .4393 & 1.80 & 0.078 & 43.93 & -.1218 & -0.43 & 0.672 & \\
\hline Gastos Com. & .3884 & 1.30 & 0.200 & & 1.24 & 5.44 & 0.000 & 124 & 1.633 & 4.60 & 0.000 & 163.3 \\
\hline Gastos Pub. & -.0236 & -0.14 & 0.893 & - & -.1219 & $-1 . .05$ & 0.300 & - & -.1937 & -1.41 & 0.164 & - \\
\hline Alim. & 1.079 & 1.41 & 0.167 & & .5320 & 1.05 & 0.300 & & -.1869 & -0.34 & 0.735 & \\
\hline
\end{tabular}

Fuente: Elaboración propia con Software Stata

cuadrada de los tres modelos son .3602, .6620 y .5313 para los años 2014, 2009 y 2004 respectivamente; es decir, el 36.02\%, 66.20\% y 53.13\% de las variables que integran los modelos explican la variabilidad de las unidades económicas de los años censales mencionados (tabla 2).

Los inventarios iniciales fueron el único caso con una significancia constante en los tres periodos censales. Los efectos marginales indican que, al aumentar en $1 \%$ el monto total de inventarios iniciales, disminuirán las unidades económicas en un $90.33 \%, 88.22 \%$ y $51.49 \%$ para los periodos comentados. Contrariamente sucede con los activos totales, que tuvieron significancia y una asociación positiva para el periodo 2014 y 2009, los efectos marginales indican que al ascender en
1\% la partida en cuestión, la cantidad de competidores aumenta en $98.23 \%$ y $43.93 \%$. Finalmente, otra partida no menos importante (gastos de comunicación) resultó significativa en 2009 y 2004. Las asociaciones porcentuales indican que al incrementar en $1 \%$ los gastos de comunicación, estos aumentarán la cantidad de competidores o unidades económicas en 124\% y 163.3\% respectivamente.

\section{DISCUSIÓN Y CONCLUSIONES}

El artículo aporta que los requerimientos de capital son una partida fundamental para poder competir en el sector alimentos y bebidas, tales son los casos de los inventarios iniciales y los activos. El primer elemento mencionado es un 
recurso interno de las empresas que es transformado en una ventaja competitiva, porque incide positivamente en las ventas y a su vez es sostenible. Lo anterior porque afecta negativamente a las unidades económicas establecidas, logrando con ello poder de mercado y una condicionante para permanecer en los sectores industriales analizados.

De hecho, los inventarios (activo circulante) reflejan amplias capacidades de producción, apalancamiento mediante créditos con los distintos canales de distribución y es un condicionante para comenzar a operar en los ejercicios comerciales y contables de las compañías, lo que permite reflexionar sobre su importancia en las sub-ramas de alimentos y bebidas. Bajo este supuesto, se coincide en que es un elemento de disuasión previo a la entrada de competidores.

Por otro lado, los activos fijos son recursos necesarios que tienen las empresas para producir y funcionar. La variable señalada es una ventaja competitiva porque dentro de ella hay sub-partidas como acervo total en maquinaria, inmobiliario y equipo de oficina, bienes inmuebles y equipo de transporte que colaboran en sinergia para generar dividendos (efecto en las ventas). No obstante, pareciera que son elementos que no disuaden o afecten la competencia. Los rivales pueden salvar dichas condiciones por medio de arrendamientos financieros, redes de colaboración, arrendamientos con posibilidad de compra de activos o simplemente la subcontratación de servicios logísticos, lo que nulifica su capacidad de eliminar competencia en cierta medida para las sub-ramas analizadas. Por otro lado, esta variable es positiva porque es un indicador que refleja el ingreso de competidores $y$, en consecuencia, su efecto generado; es decir, si una empresa ingresa a trabajar debe tener activos fijos.

En otro contexto, partidas como los gastos publicitarios que pueden ser un elemento para generar diferenciación o ser una condicionante para competir en un sector industrial no tiene efecto alguno en el grado de competencia y su desempeño, respecto a las ventas no es consistente en las sub-ramas industriales estudiadas. Por su parte, los gastos de comunicación que funcionarían como un recurso para establecer negocios de forma más eficiente y rápida con los clientes o integrantes de la cadena de valor es un ejemplo que incide en la productividad (ventas) pero que no reduce competidores; en otras palabras, su naturaleza es positiva porque al ingresar a competir una nueva empresa debe gastar en internet, fax, teléfono y otros servicios de comunicación. Asimismo, su consistencia sobre las variables ventas y unidades económicas es debatible respecto a los inventarios y los activos en los periodos censales examinados.

El trabajo coincide en que los recursos de la compañía son generadores de ventaja competitiva vía desempeño. De hecho, con otra metodología basada en datos censales se confirman los hallazgos de Morgan et al. (2004) y Ainuddin et al. (2007) y refutando los hallazgos de Ismail et al. (2010) quien encuentra inconsistencia en los recursos de la compañía como indicadores de ventaja competitiva.

Por otro lado, es importante delimitar el contexto de que la ventaja competitiva debe ser rara, insustituible, poco imitada es difícil plasmarlo en un sentido literal. Desde la perspectiva de esta investigación, los requerimientos de capital son vitales para levantar barreras a la entrada, porque las capacidades financieras en un ambiente donde hay presencia de oligopolios concentrados con altos niveles de diferenciación de productos, los financiamientos con distribuidores, la capacidad de entrega en distribución, logística y la producción a escala son factores difíciles de precisar en categorías como únicos, raros o poco imitables, solo se llega a entender que son cuantiosos y difícilmente salvados por competidores actuales y entrantes potenciales. 
Finalmente, se contribuye al debate respecto a la disuasión de entrada en una fase de pre-entrada de competidores, los inventarios iniciales indican desde una perspectiva general que los nuevos entrantes deben ingresar a competir un nivel elevado de inventarios para comenzar su ejercicio, coincidiendo con la postura de Spence $(1977 ; 1979)$ sobre los requerimientos de inversión para impedir la entrada en un periodo previo. Es decir, hay posibilidad de disuasión sobre nuevos entrantes.

Las limitantes del estudio radican en que los hallazgos abordan temáticas generales y no se puede particularizar fenómenos por empresa, debido a que se toman sub-ramas industriales de alimentos y bebidas, tampoco se tiene información primaria como estudios previos que permite hablar de particularidades. Sin embargo, se abren puertas en dos sentidos. La primera es profundizar en otras sub-ramas de manufacturas para analizar, si hay un mismo comportamiento similar en las variables estudiadas. La otra opción es retomar este tipo de estudio con una muestra particular de empresas para obtener resultados detallados del fenómeno.

\section{REFERENCIAS}

Ainuddin, A., Beamish, P., Hulland, J., \& Rouse, J. (2007). Resource attributes and firm performance in international joint ventures. Journal of World Business, 42(2), 47-60.

Bain, J. (1956). Barriers to new competition. Harvard University Press, Cambridge Massachusetts.

Barney, J. (1986). Strategic factor markets: expectations, luck, and business strategy. Management Science, 32(10), 1231-1241. doi: 10.1287/ mnsc.32.10.1231

Barney, J. (1991). Firm Resources and Sustained Competitive Advantage. Journal of Management, 17(1), 99-120.
Barney J. (2002). Gaining and Sustainning Competitive Advantage. Prentice-Hall: Pennsylvania.

Bellon, B. (1980). Le pouvoir financier et l'industrie en France, París, Le Seuil. En Vázquez, R. (2015). Concentración empresarial y cambio estructural: alimentos, bebidas y tabaco en México. Problemas del desarrollo, 46(180), 51-76.

Bridoux, F. (2004). A resource-based approach to performance and competition: An overview of the connections between resources and competition. IAG Working Papers Num. 110.

Censo Económico Industrial, INEGI. (2014, 2009 y 2004). En http://www.beta.inegi.org.mx/app/ saic/ (Consultado, Enero 2018).

Conner, K. (1991). A Historical Comparison of Resource-Based Theory and Five Schools of Thought Within Industrial Organization Economics: Do We Have a New Theory of the Firm? Journal of Management, 17(1),121-154.

Carlton, D. \& Perloff, J. (1994). Modern industrial organization. Harper-Collins College Publishers, New York.

Dierickx, I. \& Cool, K (1989). Asset Stock Accumulation and Sustainability of Competitive Advantage. Management Science 35(12),1504-1513.

Falsaw R., Glaister, K., \& Ekrem T (2006). Evidence on formal strategic planning and Company performance. Management Decision, 44(1), 9-30.

Foss, N. (1998). The Resource-Based Perspective: An Assessment and Diagnosis of Problems. Scandinavian Journal of Management, 14(3), 133-149.

Eisenhardt, K. \& Brown, S. (1998). Competing on the edge: Strategy as structured chaos. Harvard Business School Press, Boston.

Grant, R. (1991). The Resource-Based Theory of Competitive Advantage: Implications for Strategy Formulation. California Management Review, 33(3), 114-135.

Gímenez, C., \& Ventura, A. (2002). Supply chain management as a competitive advantage in the Spanish grocery sector. Published Working Paper. No. 
2, 04/2002, Barcelona, Spain: Universitat Pompeu Fabra' (UPF).

Greene, W. (2012). Econometric Analysis. Pearson, Nueva York.

Ismail, A., Rose, R., Abdullah, H., \& Uli, J. (2010). The relationship between organizational competitive advantage and performance moderated by the age and size of firms. Asian Academy of Management Journal, 15(2), 157-173.

Ketokivi, M. \& Schroeder, R. (2004). Manufacturing practices, strategic fit and performance: A routine-based view. International Journal of Operations \& Production Management, 24(2), 171-191.

Leff, N. (1974). El espíritu de empresa y la organización industrial en los países menos desarrollados: "Los grupos". El Trimestre Económico, Fondo de Cultura Económica, 41(163), 521-541.

Ma, H. (1999). Anatomy of Competitive Advantage: A select framework. Management Decision, 37(2), 709-718.

$\mathrm{Ma}, \mathrm{H}$. (2000). Competitive advantage and firm. Competitiveness Review, 10(2),15-32.

Majeed, S. (2011). The impact of competitive advantage in organizational performance. European Journal of Businness and Management, 3(4), 191-196.

Morgan, N., Kaleka, A., \& Katsikeas, C. (2004). Antecedents of Export Venture Performance: A Theoretical Model and Empirical Assessment. Journal of Marketing, 68(2), 90-108.

Newbert S. (2007). Empirical research on the resource-based view of the firm: an assessment and suggestions for future research. Strategic Management Journal, 28(2), 121-146.

Neely, A. (2005). The evolution of performance measurement research: Developments in the last decade and a research agenda for the next. International Journal of Operations and Production Management, 25(12), 1264-1277.

Peteraf, M. (1993). The Cornerstones of Competitive Advantage: A Resource-Based View. Strategic Management Journal, 14(3), 179-191.
Peteraf, M. \& Barney, J. (2003). Unraveling the resource-based tangle. Managerial and Decision Economics, 24(4), 309-323.

Porter, M. (1980). Competitive Strategy: Techniques for Analyzing Industries and Competitors. The Free Press: New York.

Porter, M. (1985). Competitive Advantage: Creating and Sustaining Superior Performance. The Free Press: New York.

Porter, M. (1991). Towards a dynamic theory of strategy. Strategic Management Journal, 12(2), 95-117.

Precisiones del censo económico. (2014). Recuperado de http://www.inegi.org.mx/est/cubos/saic/ saic_historico_precisiones.pdf

Raduan C., Jegak U., Haslinda, A., \& Alimin I. (2009). Management, Strategic Management Theories and the Linkage with Organizational Competitive Advantage from the Resource-Based View. European Journal of Social Sciences, 11(3), 402-417.

Ray, G., Barney, J., \& Muhanna, W. A. (2004). Capabilities, business processes, and competitive advantage: Choosing the dependent variable in empirical tests of the resource-based view. Strategic Management Journal, 25(2), 23-37.

Rendon A. y Morales A. (2008). Grupos Económicos en la Industria de Alimentos: Las estrategias de Gruma. Nueva Época, 21(57), 87-112.

Ríos, E., Olvera O., y González, J. (2016). La importancia de la diferenciación en una estructura de mercado oligopólica y su efecto en los precios: El caso de la leche empacada (2015). En E. Monreal, M. Paz, M. Aguilar, C. De Luna, A. Saldivar, R. Medina. (Eds.). Investigación Aplicada de las Universidades Politécnicas en el marco del XV Aniversario del Subsistema. Edición Universidad Politécnica de Aguascalientes. Aguascalientes, México.

Ríos, E., López, P., \& Garzón, G. (2016). Las limitaciones en un oligopolio diferenciado bajo un esquema Stackelberg y su efecto en los precios en México: El caso de los refrescos (2015). Tiempo Económico, 32(11), 23-41. 
Ríos E., Conraud E., y Cansino D. (2018). Are All Strategies of Product Differentiation and Positioning Effective Through Consumer Pricing? The Case of Packaged Ice Cream in Mexico: 2017. European Journal of Business Management, 10(11), 22-34.

Rumelt, R. (1984). Towards a strategic theory of the firm. In R. Lamb, (Ed.). Competitive Strategic Management. Prentice-Hall, Englewood Cliffs (NJ).

Rumelt, R. (1991). How much does industry matter? Strategic Management Journal 2(12), 167-185.

Sánchez, R. (1993). Strategic Flexibility, firm organization and managerial work in dynamic markets: A strategic options perspective in Shiripastava $P$ y Huff J. Advances in Strategic Management, Jai Press, Greenwich CT.

Sánchez, R. (1995). Strategic Flexibility in product competition. Strategic Management Journal, 16(2),135-159.

Sampler, J. (1998). Redefining Industry Structure for the Information Age. Strategic Management Journal, 19(4), 343-355.

Sekaran, U. (2005). Research Methods for Business with SPSS 13. O Set. John Wiley \& Sons, Incorporated: Londres.

Schmalensee, R. (1985). Do Market Differ Much? The American Economic Review, 75(3), 341-351.

Schoemaker, P. (1990). Strategy, complexity, and economic rent. Management Science, 36(10), 1178-1192.

Spence, M. (1977). Entry, Capacity, Investment and Oligopolistic Pricing. Bell Journal of Economics, 8(2), 534-544.

Spence, M. (1979). Investment Strategy and Growth in a New Market. Bell Journal of Economics, 10(1), 1-19.

Spender, J. (1996) Making Knowledge the Basis of a Dynamic Theory of the Firm. Strategic Management Journal, Special Issue(7), 45-62.

Stalk, G. (1990). Time the next source of competitive advantage. Harvard Business Review, 68(4), 41-51.
Tarzijan, J. \& Paredes J. (2006). Organización Industrial para la estrategia empresarial. Pearson: Ciudad de México.

Teece, D., Pisano, G., \& Shuen, A. (1997). Dynamic capabilities and strategic management. Strategic Management Journal, 18(7), 509-533.

Vázquez, R. (2015). Concentración empresarial y cambio estructural: alimentos, bebidas y tabaco en México. Problemas del desarrollo, 46(180), 51-76.

Wang, Y. \& Lo, P. H. (2003). Customer-focused performance and the dynamic model for competence building and leveraging: A resource-based view Journal of Management. Journal of Management Development, 22(6), 483-526.

Wernerfelt, B. \& Montgomery, C. (1986). What is an attractive industry? Management Science, 32(10), 1223-1230.

Williamson, O. (1991). Strategizing, economizing, and economic organization. Strategic Management Journal, 1(12), 75-94.

Recibdio:26 de febrero de 2018

Aceptado: 08 de mayo de 2019 


\section{ANEXO}

- Cálculo de los efectos porcentuales de una variable logarítmica

Según Greene (2012) ², un modelo con variable dependiente logarítmica y regresores dummy, se representa así:

$$
\ln Y=\beta 1+\beta 2 x+\beta 3 d+\varepsilon
$$

El coeficiente de la variable dummy, $d$ indica un cambio multiplicativo de la función. El porcentaje de cambio en $E[y \mid x, d=1]$ asociado a un cambio en $d$ es:

$$
\begin{gathered}
\%((\Delta E[y \mid x, d]) / \Delta d)=100 \%\{(E[y \mid x, d=1]-E[y \mid x, d=0]) /(E[y \mid x, d=0])\} \\
=100 \%\{(\exp (\beta 1+\beta 2 x+\beta 3 E[\exp (\varepsilon)]-\exp (\beta 1+\beta 2 x) E[\exp (\varepsilon)) /(\exp (\beta 1+\beta 2 x) E[\exp (\varepsilon)])\} \\
=100 \%[\exp (\beta 3)-1]
\end{gathered}
$$

- Pruebas de las regresiones

Pruebas de las regresiones que tienen a la variable ventas como dependiente (2014, 2009 y 2004).

\section{NORMALIDAD}

\begin{tabular}{|c|c|c|c|c|c|c|c|c|}
\multicolumn{4}{|c|}{ Shapiro-Wilk Test } & \multicolumn{4}{c|}{ Shapiro-Wilk Test } & \multicolumn{4}{c|}{ Shapiro-Wilk Test } \\
\hline Variable & Obs & Prob. $>$ Z & Variable & Obs & Prob. $>$ Z & Variable & obs & Prob. $>$ Z \\
\hline$R$ & 51 & 0.2906 & $R$ & 52 & 0.8276 & r & 50 & 0.7242 \\
\hline
\end{tabular}

\section{HOMOCEDASTICIDAD}

\begin{tabular}{|c|c|c|c|c|c|c|c|c|c|c|c|}
\hline \multicolumn{10}{|c|}{ White Test (Cameron \& Trivedi decomposition) } \\
\hline Recurso & Chi 2 & Df & $p>z$ & Recurso & Chi 2 & df & $p>z$ & Recurso & Chi 2 & df & $p>z$ \\
\hline Het. & 24.53 & 19 & 0.1764 & Het & 24.84 & 19 & 0.1659 & Het & 21.01 & 19 & 0.3356 \\
\hline
\end{tabular}

\section{ESPECIFICACIÓN}

\begin{tabular}{|c|c|c|c|c|c|}
\multicolumn{5}{|c|}{ Test de Ramsey } \\
\hline F (3, 42) & 2.32 & $\mathbf{F}(3,43)$ & 1.00 & $\mathbf{F}(3,41)$ & 1.49 \\
\hline Prob $>F$ & 0.0893 & Prob $>$ F & 0.4021 & Prob $>F$ & 0.2414 \\
\hline
\end{tabular}

${ }^{2}$ William G. Econometric Analysis, Pearson, New York, 2012 
COLINEALIDAD

\begin{tabular}{|c|c|c|c|c|c|c|c|c|}
\hline \multicolumn{7}{|c|}{ Prueba VIF } \\
\hline Variable & VIF & $1 /$ VIF & Variable & VIF & $1 / F$ & Variable & VIF & $1 /$ VIF \\
\hline Act. Fijos & 8.91 & 0.1122 & Act. Fijos & 5.33 & 0.1875 & Act. Fijos & 5.34 & 0.1674 \\
\hline Inv. Inicial & 5.99 & 0.1670 & Inv. Inicial & 4.8 & 0.2083 & Inv. Inicial & 3.02 & 0.1874 \\
\hline Gastos de Com. & 5.76 & 0.1737 & Gastos de Com. & 4.19 & 0.2384 & Gastos de Com. & 5.97 & 0.3311 \\
\hline Gastos Pub. & 3.07 & 0.3252 & Gastos Pub. & 2.99 & 0.335 & Gastos Pub. & 3.19 & 0.3132 \\
\hline Alimentos & 1.36 & 0.7351 & Alimentos & 1.18 & 0.8487 & Alimentos & 1.09 & 0.9136 \\
\hline Mean VIF & 5.02 & & Mean VIF & 3.70 & & Mean VIF & 3.72 & \\
\hline
\end{tabular}

Pruebas de las regresiones que consideran a la variable unidades económicas como dependiente (2014, 2009, 2004).

\begin{tabular}{|l|c|c|c|c|c|c|c|c|}
\multicolumn{9}{c|}{ NORMALIDAD } \\
\multicolumn{4}{|c|}{ Shapiro-Wilk Test } & \multicolumn{3}{c|}{ Shapiro-Wilk Test } & \multicolumn{3}{c|}{ Shapiro-Wilk Test } \\
\hline Variable & obs & Prob. $>$ Z & Variable & Obs & Prob. $>$ Z & Variable & obs & Prob. $>$ Z \\
\hline$r$ & 51 & 0.7927 & $R$ & 52 & 0.1525 & $r$ & 50 & 0.0259 \\
\hline
\end{tabular}

\section{HOMOCEDASTICIDAD}

\begin{tabular}{|c|c|c|c|c|c|c|c|c|c|c|c|c|}
\multicolumn{10}{c|}{ White Test (Cameron \& Trivedi decomposition) } \\
\hline Recurso & Chi 2 & $\mathrm{df}$ & $\mathrm{p}>\mathrm{z}$ & Recurso & Chi 2 & $\mathrm{df}$ & $\mathrm{p}>\mathrm{z}$ & Recurso & Chi 2 & $\mathrm{df}$ & $\mathrm{p}>\mathrm{z}$ \\
\hline Het. & 40.73 & 19 & 0.0026 & Het & 18.48 & 19 & 0.4907 & Het & 24.69 & 19 & 0.1709 \\
\hline
\end{tabular}

ESPECIFICACIÓN
Test de Ramsey
\begin{tabular}{|c|c|c|c|c|c|}
\hline$F(3,42)$ & 2.32 & $F(3,43)$ & 1.00 & $F(3,41)$ & 1.49 \\
\hline Prob $>F$ & 0.0893 & Prob $>F$ & 0.4021 & Prob $>F$ & 0.2414 \\
\hline
\end{tabular}

\section{COLINEALIDAD}

\begin{tabular}{|c|c|c|c|c|c|c|c|c|}
\hline \multicolumn{7}{|c|}{ Prueba VIF } \\
\hline Variable & VIF & $1 /$ VIF & Variable & VIF & $1 / F$ & Variable & VIF & $1 /$ VIF \\
\hline Act. Fijos & 8.91 & 0.1122 & Act. Fijos & 5.33 & 0.1875 & Act. Fijos & 5.34 & 0.1674 \\
\hline Inv. Inicial & 5.99 & 0.1670 & Inv. Inicial & 4.8 & 0.2083 & Inv. Inicial & 3.02 & 0.1874 \\
\hline Gastos de Com. & 5.76 & 0.1737 & Gastos de Com. & 4.19 & 0.2384 & Gastos de Com. & 5.97 & 0.3311 \\
\hline Gastos Pub. & 3.07 & 0.3252 & Gastos Pub. & 2.99 & 0.335 & Gastos Pub. & 3.19 & 0.3132 \\
\hline Alimentos & 1.36 & 0.7351 & Alimentos & 1.18 & 0.8487 & Alimentos & 1.09 & 0.9136 \\
\hline Mean VIF & 5.02 & & Mean VIF & 3.70 & & Mean VIF & 3.72 & \\
\hline
\end{tabular}

